http://jmscr.igmpublication.org/home/ ISSN (e)-2347-176x ISSN (p) 2455-0450 crossref DOI: https://dx.doi.org/10.18535/jmscr/v8i8.28

\title{
Nothing is Permanent but Sometimes Changes happen...............B iomolecular Implication of Novelcoranavirus to Humanbeing...
}

\section{Authors \\ Dr Subham Kumari', Dr Sonal Sachin², Dr Ajay Kumar Bharti", Dr Anuradha Kumari ${ }^{4}$, Dr Rinky Sinha ${ }^{5}$}

${ }^{1}$ BDS, Buddha Institute of Dental Sciences \& Hospital, Patna, Fellow in Academy of General Education, Manipal Academy of Higher Education, Manipal, Practicing in Cosmetica-The total Dentofacial Solutions, Patna (Bihar)

${ }^{2}$ BDS Buddha Institute of Dental Sciences \& Hospital, Patna, Patna (Bihar)

${ }^{3}$ BDS, Buddha Institute of Dental Sciences \& Hospital, Patna, Practicing in Bhagalpur Dental Care, Bhagalpur (Bihar)

${ }^{4}$ BDS, Buddha Institute of Dental Sciences \& Hospital, Patna, Private Practitioner, Patna (Bihar)

${ }^{5}$ BDS, Buddha Institute of Dental Sciences \& Hospital, Patna, Practicing in Kritika Dental Clinic, Patna

(Bihar), India

\begin{abstract}
In this article my aim is to, summarize the effective collection of the host factors co-operated \& indicating zonal pathways activated during human novelcoranavirus infection, and its whole biomolecular relation, get evoking on Human CoV-infection with inducing stress response, autophagy, apoptosis, and innate immunity.

Human noval coronavirus (HCoV) infection which causes respiratory diseases that undergoes mild to severe results. In Past 10-15 years, in the investigation and observation we find inception of 2 zoonosis which is huge pathogenic Human CoVs.

- Severe Acute respiratory syndrome coronavirus (SARS-CoV)

- Middle East respiratory syndrome coronavirus (MERS-CoV)

Reduplication of Human CoV is modulated by a multiplicity of the host factors that instigate sudden rebuilding in inner cellular structures and anatomy .exhilarating of censorious indicating pathways during Human CoV infection regulates the stipulates of antiviral immune responses and donate to the pathological process of Human CoV. Modern history and collection of previous facts have begun to disclose some elemental aspects of the serpentine HCoV-host interrelation and

Stephania tetrandra and the other coupled species of Menispermaceae are the prime sources of the bisbenzylisoquinoline alkaloids tetrandrine (TET), fangchinoline (FAN), and the cepharanthine (CEP). Even though the pharmacological effects of those compounds includes anticancer and anti-inflammatory action, antiviral results of those compounds in case of human coronavirus (HCoV) remains uncertain. The antiviral effects of TET, FAN, and CEP and to illuminate the principal mechanism in Human CoV-OC43infected MRC-5 human lung cells. Those compounds remarkably prohibited virus-induced apoptosis at the primary stage of virus infection. TET, FAN, and CEP treatment adequately repressed the reduplication of Human CoV-OC43 along with repressed viral $S$ and $N$ protein appearance. The virus-evoked host
\end{abstract}


responses was decreased by combination treatment as differentiate with the vehicle command. Taken simultaneously these detects illustrate that TET, FAN, and CEP are prospective universal antiviral agents for the precaution and treatment of HCoV-OC43 infection.

Implementing RNA extricate from cells contaminated by SARS CoV as an effective control, those analysis were appear to have a magnetic range of at least 7 orders of expance (2x10-4-2000 TCID50/reaction). Implementing DNA plasmids as productive grade, the diagnosis control of those analysis were establish to be below ten copies / reaction. All adverse control sample were adverse in the analysis. Specimen from 2 2019-novel CoV-contaminated patients were assured in the tests. by this we got In the combined conclusion The confirmed analysis can establish a quick diagnosis of 2019noval-CoV in humanbeing samples, thereby permitting quick recognition of patients.

Keywords: SARS-CoV, MERS-CoV.

\section{Introduction}

From 2002 to 2003, SARS-CoV contaminated 8 thousand people, with a mortality rate of $\sim 10 \%$. in view of 2012, MERS-CoV has contaminated more than 1,7 hundred people, accompanied by mortality rate of $\sim 36 \%$. in view of 2013 , porcine epidemic diarrhea coronavirus (PEDV) has scrape throughout the US, give rise to an almost $100 \%$ mortality rate in piglets and eliminate more than $10 \%$ of America's total pig populations in less than one year . Usually, $\mathrm{CoV}$ cause rampant respiratory, GI, and CNS. Diseases in humanbeing and other animals, frightening humanbeing health and prompting economic loss.

\section{Features}

$\mathrm{CoV}$ belongs to the family Coronaviridae in the order Nidovirales.

These classified into 4 genera:

Alphacoronavirus $(\alpha-\mathrm{CoV})$

Betacoronavirus, $(\beta-\mathrm{CoV})$

Gammacoronavirus, $(\gamma-\mathrm{CoV})$

Deltacoronavirus $(\delta-\mathrm{CoV})$

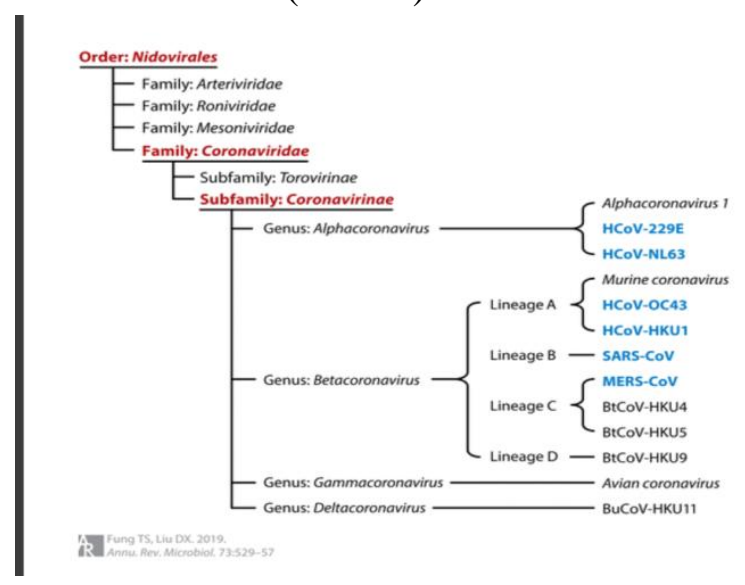

Including them, $\alpha$ and $\beta-\mathrm{CoV}$ infects mammals, $\gamma-\mathrm{CoV}$ infects avian species, and $\delta$-CoV infects both mammals and avian species. Prototypical $\alpha$ CoV includes HCoV-NL63, TGEV, PEDV, PRCV. Prototypical $\beta-\mathrm{CoV}$ includes SARS-CoV, MERS-CoV, bat coronavirus HKU4, mouse hepatitis coronavirus (MHV), bovine coronavirus (BCoV), and human coronavirus OC43. Prototypical $\gamma$-and $\delta$-CoV includes avian infectious bronchitis coronavirus (IBV) and porcine deltacoronavirus (PdCV), separately. CoV are Big, enfold, positive-stranded RNA viruses

\section{Clinical implication and role of spike protein}

In Nov 2002, a viral respiratory disease firstly immersed in south most China and rapidly develop to other countries, dominant to over 8 thousand confirmed cases at the ending of the epidemic in June 2003, with a death rate of $\sim 9.6 \%$. The etiological agents were identified as SARS-CoV, a zoonotic $\beta-\mathrm{CoV}$ drived in horseshoe bats that slowly transformed to infect the intermediate host palm civet and finally humans. following an incubation period of 4-6 days, SARS patients develops flu-like symptoms and pneumonia, which in critical cases leads to mortal respiratory failure and ARDS. Even though SARS-CoV contaminates numerous organs and causes systemic disease, symptoms actually magnify as the virus is cleared, recommending that deviant immune responses may underlying the pathogenesis of SARS-CoV. Inspite of no cases of SARS have been announced since 2004, a rich gene pool of bat SARS-related $\mathrm{CoV}$ was 
discovered in a cave in Yunnan, China, emphasize the necessary to prepare for future reappearance. The RNA genome of SARS-CoV-2 has 29,811 nucleotides, encoding for 29 proteins, as per studying these different components of the virus, as well as how they interact with our cells, is already yielding some clues, but much remains to be learned, Ott says.

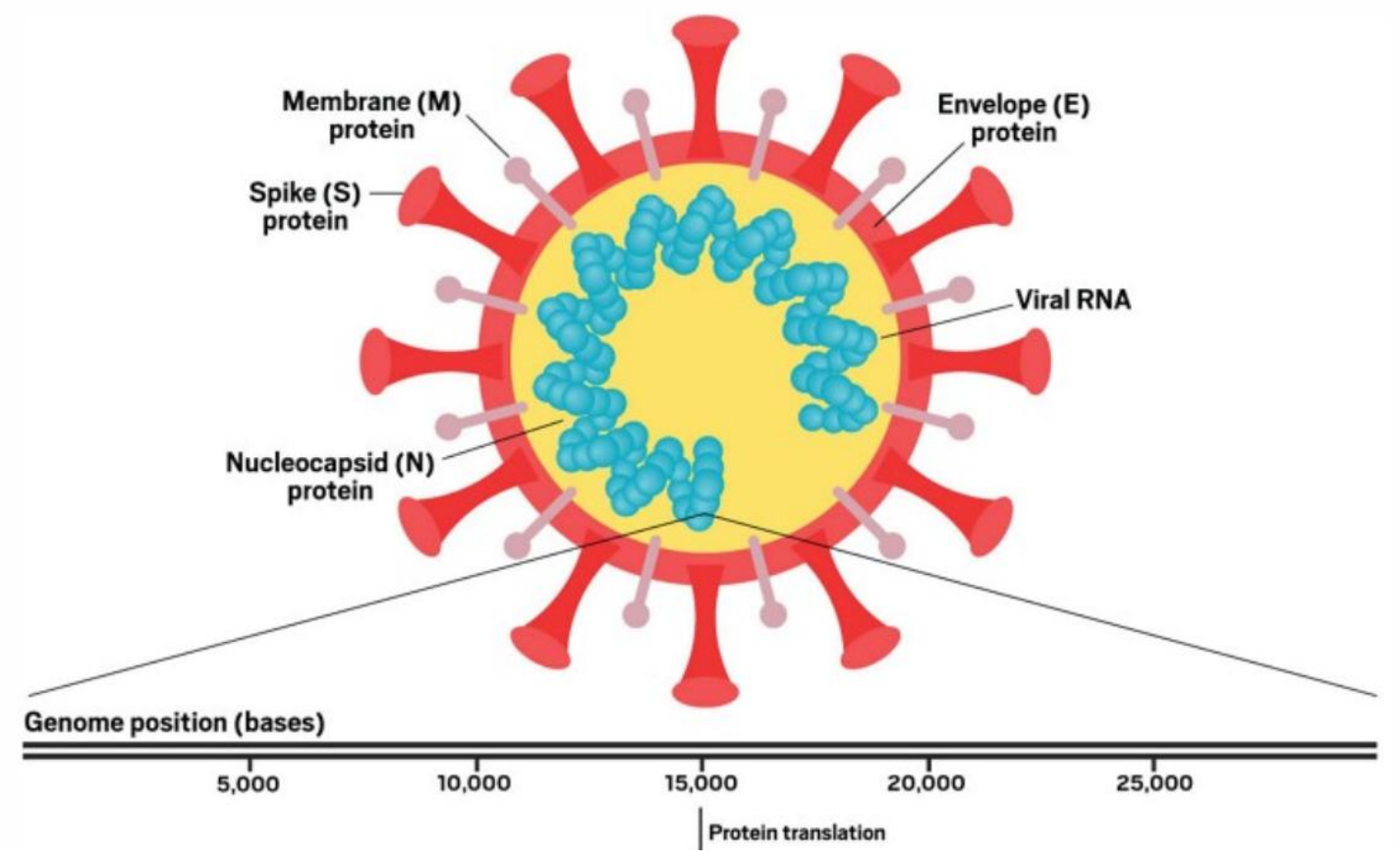

\section{Credit: Adapted from Nature and bioRxiv}

SARS-CoV-2 has four structural proteins (top): the $\mathrm{E}$ and $\mathrm{M}$ proteins, which form the viral envelope; the N protein, which binds to the virus's RNA genome; and the S protein, which binds to human receptors. The viral genome consists of more than 29,000 bases and encodes 29 proteins (bottom). The nonstructural proteins (blue) get expressed as two long polypeptides, the longer of which gets chopped up by the virus's main protease. This group of proteins include the main protease (NSP5) and RNA polymerase (NSP12).

\section{The spike}

Coronaviruses are named for the crown of protein spikes covering their outer membrane surface. Early work on the novel coronavirus has focused on these spike proteins - also called S proteinsbecause they are the keys that the virus uses to enter host cells. In both SARS-CoV and SARS$\mathrm{CoV}-2$, the $\mathrm{S}$ protein binds to a receptor called angiotensin converting enzyme 2 (ACE2) to hack its way into host cells.
At the amino acid level, the spike proteins on SARS-CoV-2 are about $80 \%$ identical to those on SARS-CoV. "The residues which interact with ACE2 are conserved, compared to [the first] SARS, but the residues in between are different, and there are also some insertions," says Rolf Hilgenfeld, a structural biologist who studies coronaviruses at the University of Lübeck.

Studies so far suggest that the new virus's spike proteins bind to ACE2 significantly more strongly than those of SARS-CoV. "That is probably one of the reasons it spreads more easily and is more infectious," Hilgenfeld says.

The other 28 Of the 29 SARS-CoV-2 proteins, four make up the virus's actual structure, including the $\mathrm{S}$ protein. One group of the other 25 coronavirus proteins regulates how the virus assembles copies of itself and how it sneaks past the host immune system. These so-called nonstructural proteins are expressed as two huge polyproteins that are then cleaved into 16 smaller proteins. An enzyme called the main protease, which performs 11 of those cleavages, is also a highly promising drug target. Hilgenfeld and his 
colleagues recently reported the structure of the main protease and identified an inhibitor that can block it.

\section{Conclusion}

Such a primitive spike might function incompetently because the inherited virus would have to spread nonspecifically to the nearest vicinity of target cells so that membrane fusion could take place. Later, the spike would enlarge a galectin- like S1-NTD through gene capture, which direct intensify its productivity in interposing virus entry. Next, the spike direct evolve an S1CTD through gene duplication of its S1-NTD or any other mechanism, farther intensifying its receptor identification function. Interpretation the morphology and physiology of CoV spikes and their development can increase our interpretation of the source of viruses and the developmental relationship between viruses and host cells.

We evaluated our assays using a panel of positive and negative control samples. The assays were found to be sensitive and specific to only sarbecoviruses. We further used respiratory specimens from patients infected by 2019-nCoV to demonstrate the potential use of these tests. The tested clinical samples were different in nature (sputum vs. throat swab) and they were collected at different onset times (day 5 vs. day 3). Thus, it is premature to use these data to determine the viral replication kinetics in humans. Further systematic investigations on clinical specimens collected from 2019-nCoV-infected patients at different post-onset time points will be needed. Nonetheless, the results demonstrated the clinical value of these respiratory samples for molecular detection of 2019-nCoV. In addition, the $\mathrm{N}$ gene RT-PCR assay was found to be more sensitive in detecting 2019-nCoV RNA in the studied clinical samples. It is possible that these clinical samples might contain infected cells expressing subgenomic mRNA (16), resulting in more $\mathrm{N}$ gene copies in the samples.

\section{Reference}

1. What do we know about the novel coronavirus's 29 proteins? https://cen.acs.org/biologicalchemistry/infectious-disease/know-novelcoronaviruss-29-proteins/98/web/2020/04

2. Morphology, physiology, and development of Coronavirus Spike Proteins https://www.ncbi.nlm.nih.gov/pmc/articles /PMC5457962/

3. An EVOLVING CASE OF RESPIRATORY DISTRESS

○ Michael ullo MD ,Gregory Sugalski MD

- Journal of the American College Of Emergency

○ Physicians Open

4. Ida guidelines https://www.ida.org.in/pdf/IDA_Recomme ndations_for_Dental_Professionals_on_the _Coronavirus_Threat.pdf

5. Aerosol splatter and dentistry https://www.ncbi.nlm.nih.gov/pubmed/151 27864

https://www.ncbi.nlm.nih.gov/pubmed/182 47451

6. Epidemic Diseases Act 1897 - India https://en.wikipedia.org/wiki/Epidemic_Di seases_Act, 1897

7. Indians Having regional and Regional Advantage.

https://theprint.in/india/indians-seem-tohave-genetic-and-regional-advantages-infight-agains tcorona virus/393939/5. 